\title{
A Novel Fuzzy CMeans-Based Clustering Scheme for Wireless Sensor Networks
}

\author{
${ }^{1}$ Smira Barzegari, ${ }^{2}$ Mohammad Masdari \\ ${ }^{1}$ Department of Computer Engineering, Urmia Branch, Islamic Azad University, \\ Urmia, Iran, M.Masdari@Iaurmia.ac.ir \\ 2 Department of Computer Engineering, Urmia Branch, Islamic Azad University, \\ Urmia, Iran, \\ barzegari.samira@gmail.com
}

\begin{abstract}
WSNs consist of many low-cost autonomous sensors that are equipped with a small battery, data processing capabilities, and short-range wireless communication interfaces. They are often deployed for monitoring and surveillance of certain phenomena of interest. In this paper, we propose a fuzzy Cmeans-based clustering scheme which improves the network lifetime by creating symmetric clusters. It improves the random process of selecting the clusterhead nodes and by creating symmetric clusters reduces the total distances of intra-cluster communications. Thus, because, sensed data are transmitted to the closer clusterheads, our proposed clustering solution can prolong the network lifetime.
\end{abstract}

Keywords: Sensor network, Cluster head, Lifetime, Energy

\section{Introduction}

A wireless sensor network or WSN is a collection of spatially distributed autonomous sensors to monitor physical or environmental conditions, such as temperature, sound, pressure. WSNs are often deployed in unattended and hostile environments and the sensors cooperatively pass their data through the WSN to a base station or sink node[1,2]. However, sensor networks have limited power and constrained processing capabilities. Therefore, designing energy efficient solutions in WSNs is a challenging issue[3].

One of the techniques that are used for decreasing the energy consumption of sensor networks is the clustering of sensors into disjoint and non-overlapping groups [4-7]. Clustering is implemented by the help of clustering algorithms that select some nodes as clusterheads based on some metric such as mobility, stability of node, energy level or etc. Each clusterhead accepts its neighboring nodes as its cluster member and tries to manage them. Also, clustereads help the sensor nodes to forward their sensed data to the sink by consuming less energy $[3,8,9]$. The clustering algorithms proposed in the literature have various objectives, for example they may provide load balancing, fault-tolerance, maximize network lifetime or connectivity. In addition, clustering has other advantages such as scalability, decreasing routing overheads, conserving communication bandwidth, stabilizing the network topology and easy topology maintenance. Moreover, clustering decreases the energy consumption of the sensor network and increases the life span of WSN.

LEACH or Low-Energy Adaptive Clustering Hierarchy is presented by Heinzelman et al in [10]. It assumes a dense sensor network of homogeneous, energy-constrained nodes, which will report their data to a sink node. It creates clusters of sensor nodes by randomly and distributedly selected sensor nodes as clusterheads. Because the sink is often far away, the clusterheads must spend significant energy for this transmission. To 
reduce this problem, LEACH utilizes randomized rotation of cluster-heads to evenly distribute the energy load among the sensors in the network [3, 11, 12]. LEACH uses localized coordination to enable scalability and robustness for dynamic networks, and incorporates data fusion into the routing protocol to reduce the amount of information that must be transmitted to the base station $[11,13,14]$. Therefore, it can decrease the energy consumption and is able to distribute energy dissipation evenly throughout the sensors to increase the networks lifetime $[11,15,16]$.

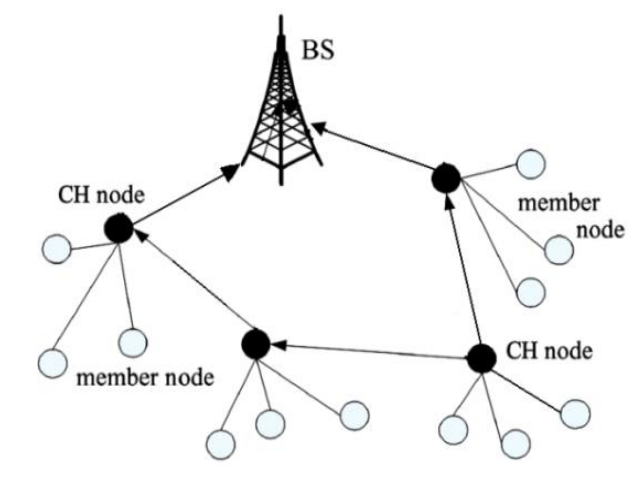

Figure 1. Clustering in Wireless Sensor Networks

To solve this problems, in this paper we propose a FCM-based clustering algorithm. This scheme produces symmetric clusters and selects nodes as clusterheads which are positioned near the center of the cluster members. By selecting such clusters, this solution tries to reduce the total distance for intra-cluster communications and this causes low power consumption in the sensor nodes. By creating symmetric clusters our solution tries to prolong the WSN's lifetime and increase the amount of data transmitted to the sink during the network lifetime.

The rest of this paper is organized as follows: Section 2 provides an overview of the LEACH protocol and its capabilities and problems, section 3 presents our proposed FCMbased clustering method and its advantages. Section 4 provides the extensive simulation results of our proposed solution and the LEACH protocol and finally section 5 provides the concluding remarks.

\section{LEACH Protocol}

LEACH is a successful clustering and routing protocol, and numerous extensions of this protocol have been proposed in the literature to enhance its performance, security and other features. This section briefly demonstrates the operations of LEACH and determines its security problems.

As Figure 2 shows, the LEACH protocol is organized in some rounds and each round is consists of set-up phase and steady-state phase. The set-up phase, itself consists of the following subphases:

- Advertisement subphase.

- Cluster setup subphase.

- Broadcast schedule subphase

In Advertisement phase, each node independently determines whether or not to become a $\mathrm{CH}$ is made by the node choosing a random number between 0 and 1 . If the number is less than a threshold T(n)?:, the node becomes a cluster-head for the current round. The threshold is set as follows: 
$T(S)=\left\{\begin{array}{ll}\left(\frac{P}{1-P\left(R \bmod \frac{1}{P}\right)}\right) & \forall \mathrm{n} \in \mathrm{G} \\ 0 & \forall n \in G\end{array}\right\}$

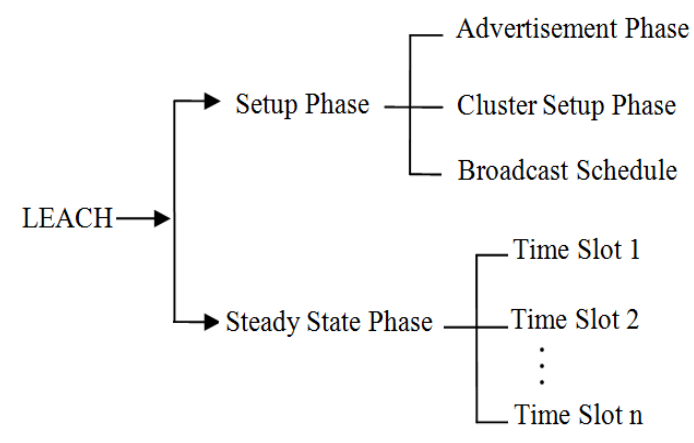

Figure 2. Phases of the LEACH Protocol

After each node independently determined to be clusterhead for the current round, it informs its neighborhood with an advertisement packet. The non- $\mathrm{CH}$ nodes pick the advertisement packet with the strongest received signal strength and in the cluster-setup phase members join to a $\mathrm{CH}$ using a CSMA protocol. In broadcast schedule subphase the $\mathrm{CH}$ knows the number of members and constructs a TDMA schedule, picks a CDMA code randomly and broadcasts this information to its members.

However, as indicated in the Figure 3, the clusters created by LEACH, often are asymmetric and clusterhead is not located in the center of member nodes. Such clusters increase the total distance between nodes and the clusterhead and this causes more energy to be consumed in the intra-cluster communications. On the other hand, when sensors want to send their data to the clusterheads, should send to clusterheads located far from them. To solve this problem, we should select some nodes as custerhead which are position near the center of the cluster members.

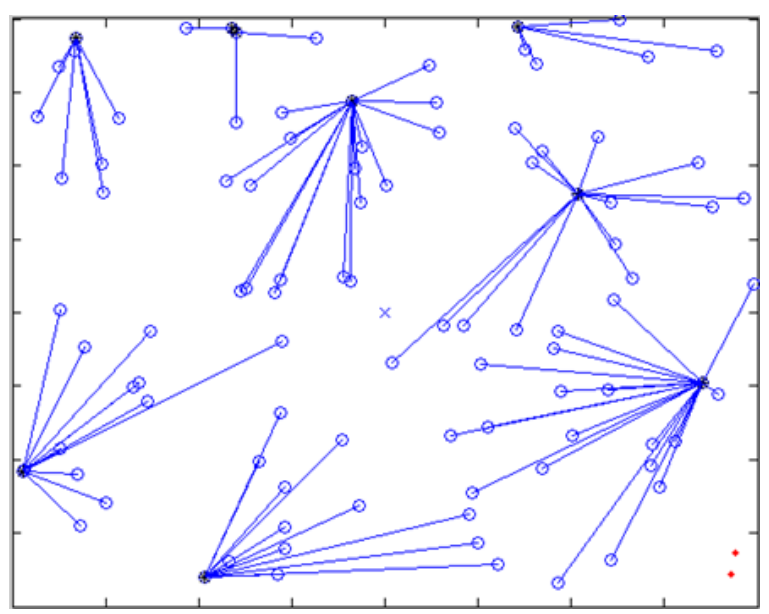

Figure 3. Clustering Using LEACH Protocol

In the next section, we present our proposed FCM-based solution to improve the LEACH protocol and increase its efficiency and network lifetime. 


\section{Fuzzy C-Means}

In fuzzy clustering data elements can belong to more than one cluster, and associated with each element is a set of membership levels. These indicate the strength of the association between that data element and a particular cluster. Fuzzy clustering is a process of assigning these membership levels, and then using them to assign data elements to one or more clusters. A widely used fuzzy clustering algorithms is the Fuzzy C-Means (FCM) Algorithm proposed by Bezdek in 1981. The FCM algorithm attempts to partition a finite collection of $n$ elements $X=\left\{x_{1}, x_{2}, x_{3}, \ldots, x_{n}\right\}$ into a collection of c fuzzy clusters with respect to some given criterion. Given a finite set of data, the algorithm returns a list of c cluster centers $C=\left\{c_{1}, c_{2}, \ldots, c_{n}\right\}$ and a partition matrix $W=w_{i, j} \in[0,1], i=1, \ldots, n, j=1, \ldots, c$, where each element $W_{i j}$ tells the degree to which element $x_{i}$ belongs to cluster $c_{j}$ and can be computed as follows:

$$
w_{i j}^{m}=\frac{1}{\sum_{k=1}^{c}\left(\frac{\left\|\mathbf{x}_{i}-\mathbf{c}_{j}\right\|}{\left\|\mathbf{x}_{i}-\mathbf{c}_{k}\right\|}\right)^{\frac{2}{m-1}}} .
$$

\section{Proposed Solution}

In our scheme, we assume that sensor nodes are randomly distributed and also are equipped with GPS and can report their position to the sink. After sink gets the position of all nodes, it can create the required number of clusters by using FCM, based on the number of network nodes. Then it broadcasts the clusterheads list to all sensors of the network. After this, the sensor nodes which are declared as clusterheads, begin to operate as clusterhead and broadcast their presence on the network. The rest of the protocol is the same as the LEACH and nodes join to the closest $\mathrm{CH}$ and $\mathrm{CH}$ broadcasts the TDM scheduling for nodes.

In our proposed solution, we use fuzzy C-Means to cluster the network nodes. By using this function we can approximately create symmetric clusters and reduce the intra-cluster communication's distance. Algorithm 1 exhibits our proposed FCM-based clustering algorithm for WSN.

\begin{tabular}{|ll|}
\hline 1. & Sensor send their location information to the \\
sink \\
2. & Sink creates a number of clusters based on \\
3. & the number of WSN nodes \\
4. & After the center of clusters are found by \\
5. & FCM, the closest node to the cluster center is \\
6. & selected as clsuerhead \\
7. & Sink broadcasts the clusterheads List to the \\
WSN \\
8. \\
9. By receiving clusterhead List, clusterheads \\
10. & Like cluster setup phase, sensors join to \\
11. & the closest clusterhead \\
12. & Each clusterheads broadcast the TDMA \\
schedule \\
13. for its members. \\
14. Like Steady state Phase in the LEACH \\
protocol \\
15. Each sensor transmit its data in its time slot \\
\hline Algorithm 1: Our Proposed FCM-based \\
Clustering Algorithm
\end{tabular}


Thus we have only modified the Advertisement phase of the LEACH protocol to select better nodes as clusterhead.

As exhibited in Figure 4, by using our proposed FCM-based solution, we can achieve more symmetric clusters and this finally, this reduces the total distance for intra-cluster communication. By using this method, our solution reduces the energy consumption of nodes and improves the network lifetime.

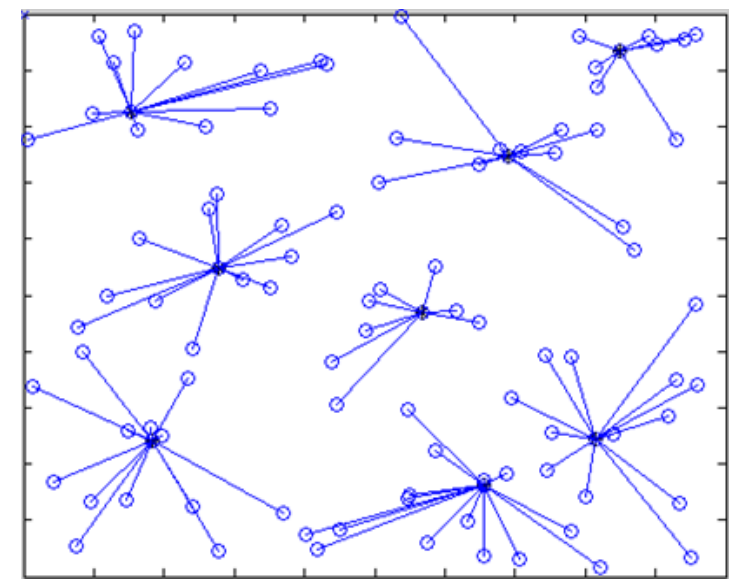

Figure 4. Clustering with our Proposed FCM-based Solution

\section{Simulation Results}

In this section, we provide the result of the extensive simulation results, which have been achieved by simulating our proposed solution and LEACH protocol in the MATLAB. We perform the simulation of our solution and the LEACH protocol in two different scenarios. In the first scenario, the simulation area is considered as $400 * 400 \mathrm{~m}$ and sink is positioned at the location of $(200,200)$. Also, 100 sensor nodes are used in the simulation area which sense the environment and try to send their data to the sink. These nodes are distributed randomly in the network and we consider $0.2 \mathrm{~J}$ for each of them. Table 1 shows the parameters which have been used in the first simulation scenario.

\begin{tabular}{|l|l|}
\hline \multicolumn{2}{|c|}{ Table 1. Simulation Parameters } \\
\hline \multicolumn{1}{|c|}{ Parameter } & \multicolumn{1}{c|}{ Value } \\
\hline Simulation Area & $(400 \times 400) \mathrm{m}$ \\
\hline Sink location & $(200 \times 200) \mathrm{m}$ \\
\hline Number of nodes & 100 node \\
\hline Initial energy & $0.2 \mathrm{~J}$ \\
\hline ETX & $50 \times 0.000000001 \mathrm{~J}$ \\
\hline ERX & $50 \times 0.000000001 \mathrm{~J}$ \\
\hline Efs & $10 \times 0.000000000001 \mathrm{~J}$ \\
\hline EDA & $5 \times 0.0000000001 \mathrm{~J}$ \\
\hline
\end{tabular}




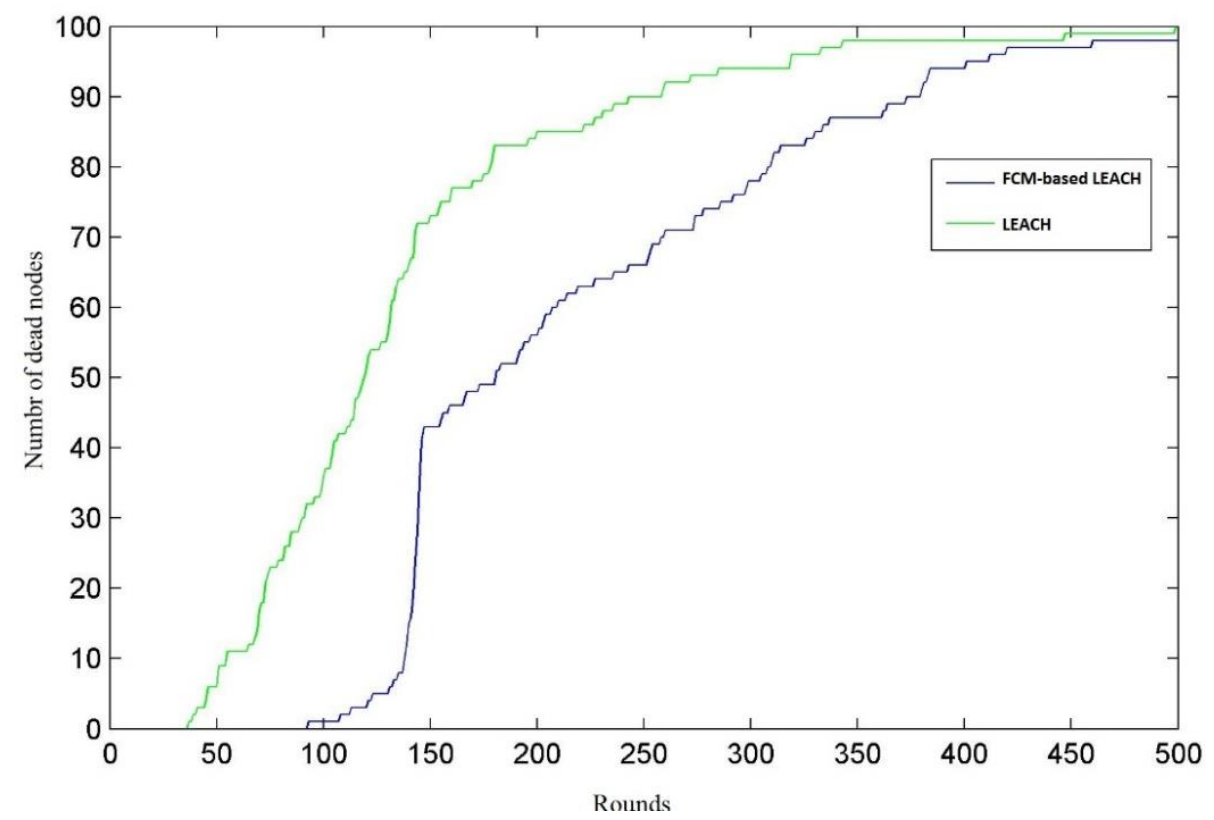

\section{Figure 5. Number of Dead Nodes in each Round of LEACH and Our Proposed Protocol}

In the first simulation scenario, we evaluate the following parameters in our proposed solution and the LEACH protocol:

- Number of Dead nodes in each round

- Average energy of sensor nodes in each round

- Total distance of transmitted data

Figure 5 shows the number of dead nodes in each round of LEACH and our proposed protocol in the first scenario. When network nodes consumes their energy and their battery deplete, we consider them as dead nodes.

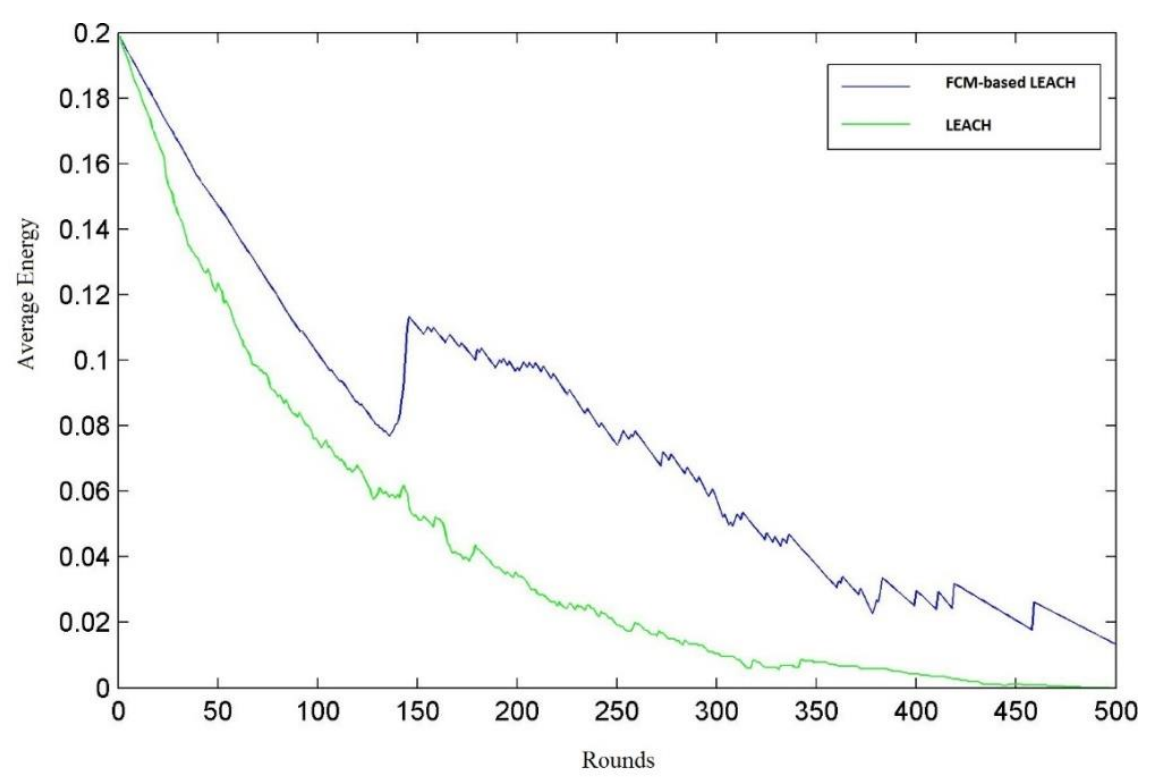

Figure 6. Average Energy of Sensor Nodes in each Round of LEACH and Our Proposed Protocol 


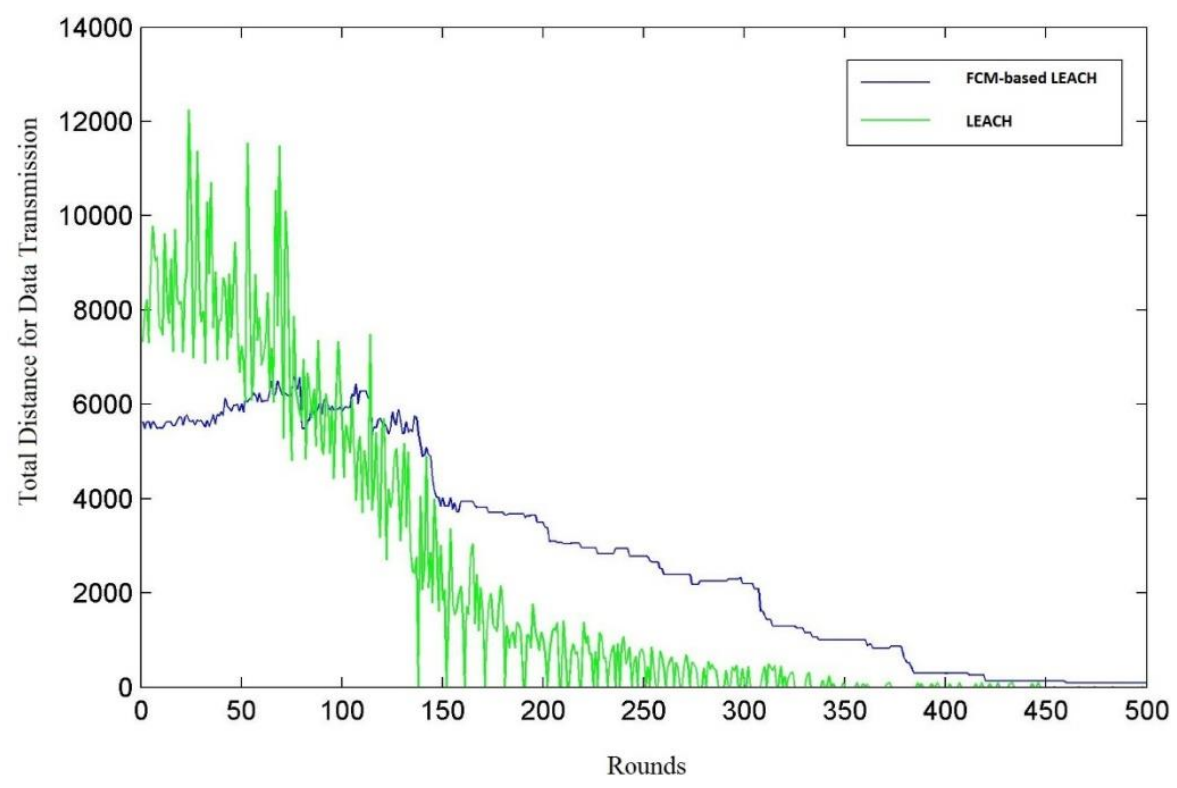

\section{Figure 7. Total Distance of Transmitted Data in LEACH and Our Proposed Protocol}

As indicated in this figure, our solution improves the network lifetime and this increase the amount sensed and transmitted by sensors. As indicated in this figure, our solution improves the First Node Die time and the first node which dies, at least lives twice as in the LEACH protocol.

Figure 6 exhibits average energy of sensor nodes in each round of LEACH and our proposed protocol in the first scenario which nodes have $0.2 \mathrm{j}$ battery power.

As we outlined before, our scheme by transmitting data to clusterheads that are near, consumes less energy and the average energy of network nodes will be higher than the LEACH protocol.

Figure 7 indicates the total distance of transmitted data in LEACH and our proposed protocol in the first scenario. As shown in this figure, our solution has less total communication distance than $\mathrm{LEACH}$, but as more rounds of the simulation are executed, our solution's total distance increases because, LEACH's nodes die sooner than our proposed solution.

Table 2 shows the parameters which have been used in the first simulation scenario. In the first scenario, the simulation area is considered as 500*500meter and sink is positioned at the location of $(0,500)$. Also, 100 nodes are considered to be in the simulation area which sense the environment and try to send data to the sink. Also, $1.5 \mathrm{~J}$ is considered for each

\begin{tabular}{|c|c|}
\hline Parameter & Value \\
\hline Simulation Area & $(500 \times 500) \mathrm{m}$ \\
\hline Sink location & $(0,500) \mathrm{m}$ \\
\hline Number of nodes & 100 node \\
\hline Initial energy & $1.5 \mathrm{~J}$ \\
\hline ETX & $50 \times 0.000000001 \mathrm{~J}$ \\
\hline ERX & $50 \times 0.000000001 \mathrm{~J}$ \\
\hline Efs & $10 \times 0.000000000001 \mathrm{~J}$ \\
\hline$\overline{E D A}$ & $5 \times 0.0000000001 \mathrm{~J}$ \\
\hline
\end{tabular}


Figure 8 indicates the average energy of sensor nodes in each round of LEACH and our proposed protocol in the second scenario. As indicated in this figure our proposed solution consumes less power for transmitting the data from sensor nodes to the sink and almost in all rounds the average of sensors' energy is maintained better than the LEACH protocol. This average is computed from the energy of the live nodes which have nonzero energy.

Thus as shown in the previous two scenarios, our solution reduces the number of dead nodes and achieves this by reducing the required energy for transmission of sensed data.

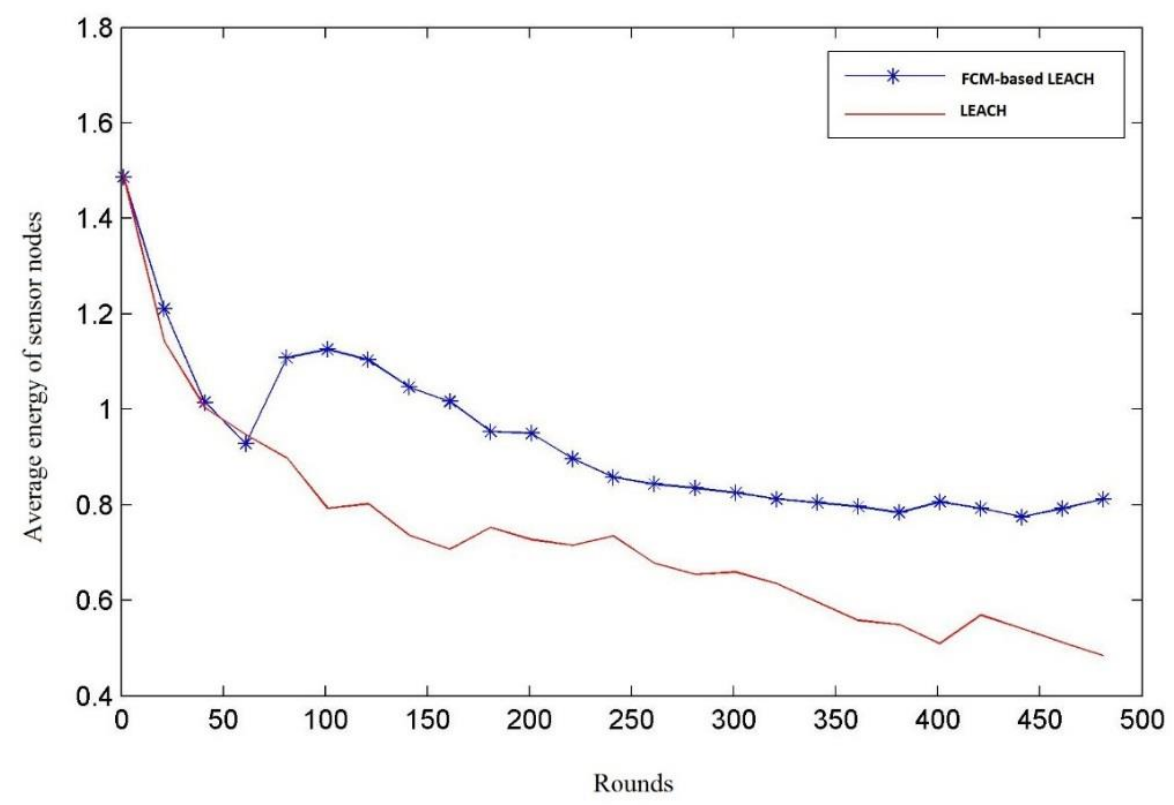

Figure 8. Average Energy of Sensor Nodes in each Round of LEACH and our Proposed Protocol

As outlined before, because of the symmetric clusters which have been produced by our scheme, our solution sends the sensed data to closer clusterheads tan the LEACH and this mitigates the energy consumption for data transmission.

\section{Conclusion}

Wireless sensor network consists of a large number of micro sensor nodes deployed in the monitoring area, and forms a self-organized network system by the wireless multi-hop communication. However, sensors in WSN have limited battery power and this limits the lifetime of network. Clustering of network nodes can be used to mitigate this problem by selecting appropriate nodes as clusterheads.

In this paper, we applied fuzzy CMeans clustering algorithm for producing symmetric clusters. By using this method we reduce the communication distance for the intra-cluster communications. We indicted that this causes reduction in power consumption in sensor nodes which result in prolonging the WSN's lifetime. Also, creating symmetric clusters improves the FND time and LND time in our proposed solution.

\section{References}

[1] M.A.M. Vieira, "Survey on wireless sensor network devices. in Emerging Technologies and Factory Automation", 2003. Proceedings. ETFA'03. IEEE Conference. 2003. IEEE.

[2] J. B.Yick, Mukherjee, and D. Ghosal, Wireless sensor network survey. Computer networks, vol. 52, no. 12, (2008), p. 2292-2330. 
[3] S. Tyagi,. and N. Kumar, A systematic review on clustering and routing techniques based upon LEACH protocol for wireless sensor networks. Journal of Network and Computer Applications, vol. 36, no. 2, (2013), p. 623-645.

[4] A.A. Abbasi, and M. Younis, A survey on clustering algorithms for wireless sensor networks. Computer communications, vol. 30, no. 14, (2007), pp. 2826-2841.

[5] V., N. Katiyar, Chand, and S. Soni, Clustering algorithms for heterogeneous wireless sensor network: A survey. International Journal of Advanced Networking and Applications, vol. 2, no. 4, (2011), p. 745-754.

[6] V. Kumar, S. Jain, and S. Tiwari, Energy efficient clustering algorithms in wireless sensor networks: A survey. IJCSI International Journal of Computer Science Issues, vol. 8, no. 5, (2011).

[7] X. Min, et al., "Energy efficient clustering algorithm for maximizing lifetime of wireless sensor networks", AEU-International Journal of Electronics and Communications, vol. 64, no. 4, (2010), pp. 289-298.

[8] O. Boyinbode, , H. Le, and M. Takizawa, "A survey on clustering algorithms for wireless sensor networks", International Journal of Space-Based and Situated Computing, vol. 1, no. 2-3, (2011), p. $130-136$.

[9] A. Chamam, and S. Pierre, A distributed energy-efficient clustering protocol for wireless sensor networks. Computers \& electrical engineering, vol. 36, no. 2, (2010), p. 303-312.

[10] Heinzelman, W.R., A. Chandrakasan, and H. Balakrishnan. Energy-efficient communication protocol for wireless microsensor networks. in System sciences, 2000. Proceedings of the 33rd annual Hawaii international conference on. 2000. IEEE.

[11] Katiyar, V., et al. Improvement in LEACH protocol for large-scale wireless sensor networks. in Emerging Trends in Electrical and Computer Technology (ICETECT), 2011 International Conference on. 2011. IEEE.

[12] Tong, M. and M. Tang. LEACH-B: an improved LEACH protocol for wireless sensor network. in Wireless Communications Networking and Mobile Computing (WiCOM), 2010 6th International Conference on. (2010), IEEE.

[13] H.M. Abdulsalam, and L.K. Kamel. W-LEACH: Weighted Low Energy Adaptive Clustering Hierarchy aggregation algorithm for data streams in wireless sensor networks. IEEE.

[14] H.M. Abdulsalam, and L.K. Kamel. W-LEACH: Weighted Low Energy Adaptive Clustering Hierarchy aggregation algorithm for data streams in wireless sensor networks. in Data Mining Workshops (ICDMW), 2010 IEEE International Conference on. (2010). IEEE.

[15] M.O. Farooq, A.B. Dogar, and G.A. Shah. MR-LEACH: multi-hop routing with low energy adaptive clustering hierarchy. in Sensor Technologies and Applications (SENSORCOMM), 2010 Fourth International Conference on. (2010). IEEE.

[16] L. Tao, Z. Qing-Xin, and Z. Luqiao. An improvement for LEACH algorithm in wireless sensor network. in Industrial Electronics and Applications (ICIEA), 2010 the 5th IEEE Conference on. (2010). IEEE. 
International Journal of Grid and Distributed Computing Vol. 9, No. 2 (2016) 